\title{
Quantum tomography of input-output processes
}

\author{
Alfredo Luis* \\ Departamento de Óptica, Facultad de Ciencias Físicas, Universidad Complutense, 28040 Madrid, Spain
}

(Received 11 April 2000; published 11 October 2000)

\begin{abstract}
We demonstrate the possibility of practical tomographic determination of arbitrary input-output transformations. It is shown that the Liouville-space formalism provides a generalization of standard tomography encompassing the reconstruction of processes as well as of quantum states.

PACS number(s): 03.65.Bz, 03.65.Db, 42.30.Wb, 42.50.Dv
\end{abstract}

\section{INTRODUCTION}

The concept of measurement has occupied a distinguished position in quantum theory from its very beginning. The standard idea of measurement deals with the procedures determining the statistics of some set of compatible observables. This original framework has been enlarged since it has been demonstrated that there are practical schemes that allow for the determination of the quantum state in which the system is $[1-6]$. Such schemes can be regarded as being measurements of the density matrix.

More recently, the idea of measurement has been further enlarged by demonstrating the possibility of experimental determinations of input-output transformations [7,8]. Among other potential applications, this might serve to ascertain the influence of external agents by disclosing the exact way in which the real process differs from the theoretically assumed one. This can be of importance for any technical application of quantum states in rapidly developing fields of research such as information processing, computation, and cryptography.

Concerning potential implementations of this reconstruction of quantum processes, it has been shown that there are measuring arrangements whose statistics provide informationally complete phase-space representations of arbitrary processes [8]. As it occurs for quantum states, there are several different representations of processes by functions $[9,10]$. Although in the ideal case all of them will provide complete information about the process, they are no longer equivalent concerning their sensitivity to unavoidable experimental errors and uncertainties.

One of the most fruitful and robust methods for reconstructing quantum states is tomography $[1,2,5,6,11]$. The quantum state can be obtained by measuring position (for trapped ions) or quadratures (for electromagnetic-field modes) after arbitrary rotations in phase space.

In this brief report, we introduce and examine the tomographic determination of transformations. To this end, in Sec. II we recall basic definitions that allow to represent arbitrary transformations by quasidistributions and characteristic functions on phase space.

Then, in Sec. III we show that one of such characteristic functions can be easily determined in practice. This can be achieved by a tomographic determination of the output states

*Electronic address: alluis@eucmax.sim.ucm.es when the input is prepared to be the eigenstates of rotated position or quadrature operators.

Finally, in Sec. IV we show that this scheme for the reconstruction of processes can be formally expressed by exactly the same equations valid for the reconstruction of quantum states. This identification can be established by using the vector representation of operators in Liouville space [12]. We think it is worth stressing the importance of this point since it implies that the practical reconstruction of processes can benefit from the well-established theoretical and experimental background of quantum-state tomography.

\section{PHASE-SPACE REPRESENTATION OF TRANSFORMATIONS}

In this section, we briefly outline the description of the most general input-output transformation and its representation by functions on phase space. The input and output degrees of freedom of interest will be represented by the Hilbert spaces $\mathcal{H}_{\text {in }}$ and $\mathcal{H}_{\text {out }}$, respectively. The corresponding quantum states are represented by the density matrices $\rho_{\text {in }}$ and $\rho_{\text {out }}$. In order to describe the most general transformation, including open as well as closed systems, we have to consider possible couplings of the systems with additional degrees of freedom that will be described by a Hilbert space $\mathcal{H}_{\text {aux }}$. Their initial state before the coupling will be described by the density matrix $\rho_{\text {aux }}$. In many situations $\rho_{\text {aux }}$ as well as the dynamical details of the coupling are unknown and out of control.

The Hilbert space $\mathcal{H}_{\text {aux }}$ is defined such that $\mathcal{H}_{\text {in }} \otimes \mathcal{H}_{\text {aux }}$ includes all degrees of freedom directly or indirectly involved in the process. In other words, $\mathcal{H}_{\text {in }} \otimes \mathcal{H}_{\text {aux }}$ is a closed system and the input-output process is necessarily described by a unitary operator

$$
U: \mathcal{H}_{\text {in }} \otimes \mathcal{H}_{\mathrm{aux}} \rightarrow \mathcal{H}_{\mathrm{out}} \otimes \mathcal{H}_{\mathrm{acc}},
$$

where $\mathcal{H}_{\text {acc }}$ is the Hilbert space needed to completely describe the image of $\mathcal{H}_{\text {in }} \otimes \mathcal{H}_{\text {aux }}$ (Fig. 1).

We assume that the total input state factorizes $\rho_{\text {in }} \rho_{\text {aux }}$. The final density matrix in the output degrees of freedom of interest $\mathcal{H}_{\text {out }}$ arises after tracing over the $\mathcal{H}_{\text {acc }}$ variables

$$
\rho_{\text {out }}=\operatorname{tr}_{\text {acc }}\left(U \rho_{\text {in }} \rho_{\text {aux }} U^{\dagger}\right)=\mathcal{U}\left(\rho_{\text {in }}\right) .
$$

Using the following decomposition for $\rho_{\text {aux }}$ : 


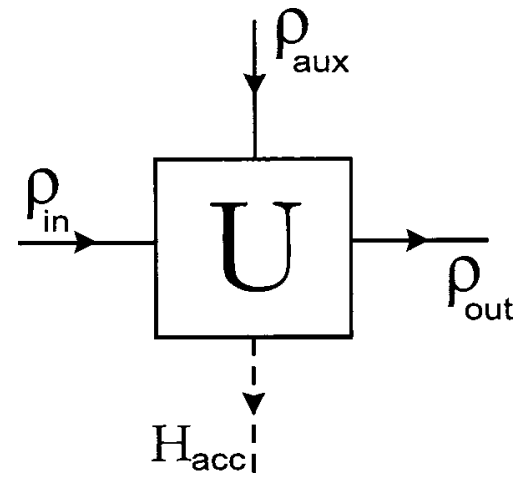

FIG. 1. Scheme illustrating the definition of the states and spaces involved in a general input-output process.

$$
\rho_{\mathrm{aux}}=\sum_{k} p_{k}\left|\psi_{k}\right\rangle\left\langle\psi_{k}\right|
$$

where $\left|\psi_{k}\right\rangle \in \mathcal{H}_{\text {aux }}$, the input-output relation can be written as

$$
\rho_{\text {out }}=\sum_{m, k} p_{k} U_{m k} \rho_{\text {in }} U_{m k}^{\dagger}
$$

where the operators $U_{m k}: \mathcal{H}_{\text {in }} \rightarrow \mathcal{H}_{\text {out }}$ are

$$
U_{m k}=\left\langle\varphi_{m}|U| \psi_{k}\right\rangle,
$$

and $\left|\varphi_{m}\right\rangle \in \mathcal{H}_{\text {acc }}$ is any family of vectors providing a resolution of the identity $I_{\text {acc }}=\Sigma_{m}\left|\varphi_{m}\right\rangle\left\langle\varphi_{m}\right|$ on $\mathcal{H}_{\text {acc }}$.

In the most general case the input and output degrees of freedom need not necessarily coincide: $\mathcal{H}_{\text {in }} \neq \mathcal{H}_{\text {out }}, \mathcal{H}_{\text {aux }}$ $\neq \mathcal{H}_{\text {acc }}$. Also $\mathcal{H}_{\text {aux }}$ and/or $\mathcal{H}_{\text {acc }}$ can be trivial so it can occur that $U: \mathcal{H}_{\text {in }} \rightarrow \mathcal{H}_{\text {out }} \otimes \mathcal{H}_{\text {acc }}$ or $U: \mathcal{H}_{\text {in }} \otimes \mathcal{H}_{\text {aux }} \rightarrow \mathcal{H}_{\text {out }}$. The lack of coincidence between input and output variables is actually the case of generalized measurements described by positiveoperator measures, where the measurement is performed on a number of outputs different from the number of relevant inputs [13]. For example, this is the case of the eight-port homodyne detector that is the basis for an operational definition of quantum phase difference [14]. In such an example $\mathcal{H}_{\text {in }}$ contains two field modes while $\mathcal{H}_{\text {out }}$ includes four field modes.

For the sake of simplicity, in what follows we will consider that $\mathcal{H}_{\text {in }}$ and $\mathcal{H}_{\text {out }}$ represent an unbounded continuous degree of freedom describable by adimensional Cartesian variables $q, p$, which in the quantum domain become operators satisfying the commutation relation $[q, p]=i$. The points of the classical phase space will be denoted by the complex variable $\alpha=(q+i p) / \sqrt{2}$, which becomes the complex amplitude operator $a$ in the quantum regime. Among other examples this includes the one-dimensional motion of a trapped ion, where $q$ and $p$ are proportional to position and momentum, and also a single mode of the electromagnetic field where $q$ and $p$ are field quadratures.

In this paper we are not interested in representing transformations by operators, such as in Eq. (2.5). Instead we will use functions that might be then suitably related to the statistics of measuring arrangements. Aside from the standard
Hilbert-space approach, there are complete formulations of the quantum theory on the classical phase space by means of which the solution of quantum-mechanical problems can be expressed in a quasiclassical form. In particular there is a family of $A \leftrightarrow W$ correspondences between operators $A$ and functions $W$ on phase space (quasidistributions) of the form $[9,10]$

$$
\begin{gathered}
W(\alpha, s)=\operatorname{tr}[A \Delta(\alpha, s)], \\
A=\frac{1}{\pi} \int d^{2} \alpha W(\alpha, s) \Delta(\alpha,-s),
\end{gathered}
$$

where the operator $\Delta(\alpha, s)$ is

$$
\Delta(\alpha, s)=\frac{1}{\pi} \int d^{2} \beta e^{s|\beta|^{2} / 2} e^{\beta^{*} \alpha-\beta \alpha^{*}} D(\beta),
$$

$D(\beta)$ is the displacement operator

$$
D(\beta)=e^{\beta a^{\dagger}-\beta^{*} a},
$$

and the parameter $s$ distinguishes between different $A \leftrightarrow W$ correspondences. As particular cases we have the $Q$ function $(s=-1)$, the $P$ representation $(s=1)$, and the Wigner function $(s=0)$.

Not only quantum states, but also processes can be fully described by functions on phase space. The input-output transformation (2.2) can be expressed as a relation between quasidistributions

$$
W_{\text {out }}\left(\alpha^{\prime}, s^{\prime}\right)=\int d^{2} \alpha \mathcal{U}\left(\alpha, s ; \alpha^{\prime}, s^{\prime}\right) W_{\text {in }}(\alpha, s) \text {, }
$$

where

$$
\mathcal{U}\left(\alpha, s ; \alpha^{\prime}, s^{\prime}\right)=\frac{1}{\pi} \operatorname{tr}\left[\Delta_{\text {out }}\left(\alpha^{\prime}, s^{\prime}\right) U \Delta_{\text {in }}(\alpha,-s) \rho_{\text {aux }} U^{\dagger}\right],
$$

and $W_{\text {in }}, W_{\text {out }}$ are the corresponding quasidistributions associated with $\rho_{\text {in }}, \rho_{\text {out }}[8,9,15]$. Formally, this function is the output for impulse inputs of the form $W_{\text {in }}(\alpha, s)=\delta\left(\alpha-\alpha_{0}\right)$. Nevertheless, not all values for $s$ allow system states having such a delta phase-space representative.

Alternatively, the input-output relation (2.2) can be also expressed as a relation between characteristic functions

$$
\chi_{\text {out }}\left(\beta^{\prime}, s^{\prime}\right)=\int d^{2} \beta \tilde{\mathcal{U}}\left(\beta, s ; \beta^{\prime}, s^{\prime}\right) \chi_{\text {in }}(\beta, s) \text {, }
$$

where $\chi_{\text {in }}, \chi_{\text {out }}$ are the characteristic functions associated with $\rho_{\text {in }}$ and $\rho_{\text {out }}$, respectively,

$$
\chi(\beta, s)=e^{s|\beta|^{2} / 2} \operatorname{tr}[\rho D(\beta)],
$$

and 


$$
\begin{aligned}
\widetilde{\mathcal{U}}\left(\beta, s ; \beta^{\prime}, s^{\prime}\right)= & \frac{1}{\pi} e^{s^{\prime}\left|\beta^{\prime}\right|^{2} / 2} e^{-s|\beta|^{2} / 2} \\
& \times \operatorname{tr}\left[D\left(\beta^{\prime}\right) U D(-\beta) \rho_{\text {aux }} U^{\dagger}\right] .
\end{aligned}
$$

Characteristic functions and quasidistributions are Fourier transform pairs so both $W(\alpha, s)$ and $\chi(\beta, s)$ provide full information about the system state. Recently, a family of generalized Wigner functions has been defined that includes $W(\alpha, 0)$ and $\chi(\beta, 0)$ as two particular cases [16]. Moreover, $\chi(\beta, s)$ is a well-behaved function even when $W(\alpha, s)$ does not exist as an ordinary function.

Therefore, the function $\widetilde{\mathcal{U}}$ in Eqs. (2.11) and (2.13) is a Fourier transform of $\mathcal{U}$ and therefore it determines completely the transformation. The use of $\widetilde{\mathcal{U}}$ or $\mathcal{U}$ is a matter of convenience and, in fact, many practical schemes directly measure characteristic functions instead of quasidistributions [4]. In particular, phase-space tomography relies on the measurement of the $s=0$ characteristic function [11].

\section{TOMOGRAPHY OF TRANSFORMATIONS}

The tomographic reconstruction of quantum states relies on the determination of the characteristic function for $s$ $=0, \chi(\beta) \equiv \chi(\beta, 0)$, which can be expressed as

$$
\chi(\beta)=\operatorname{tr}[\rho D(\beta)]=\operatorname{tr}\left(\rho e^{i \lambda q_{\theta}}\right),
$$

where the real numbers $\lambda$ and $\theta$ are defined by

$$
\beta=\frac{i}{\sqrt{2}} \lambda e^{i \theta}
$$

and the operators $q_{\theta}$ are

$$
q_{\theta}=\frac{1}{\sqrt{2}}\left(e^{-i \theta} a+e^{i \theta} a^{\dagger}\right)=q \cos \theta+p \sin \theta,
$$

which are rotated quadrature or rotated position operators. In this context it is customary to restrict $\theta$ to a $\pi$ interval $\theta$ $\in[0, \pi)$ so that $\lambda$ must take positive and negative values. The characteristic $\chi(\beta)$ can be determined in practice by measuring the operators $q_{\theta}[1,2,5,6,11]$. From Eq. (3.1) we have

$$
\chi(\beta)=\int d x e^{i \lambda x} P(x, \theta),
$$

where

$$
P(x, \theta)=\langle x, \theta|\rho| x, \theta\rangle,
$$

and $|x, \theta\rangle$ is the eigenvector of $q_{\theta}$ with eigenvalue $x$ :

$$
q_{\theta}|x, \theta\rangle=x|x, \theta\rangle
$$

In the optical case this measurement can be achieved by using balanced homodyne detection with a strong local os-

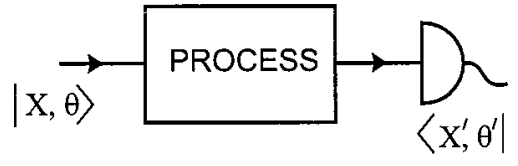

FIG. 2. Outline of the scheme for the measurement of an arbitrary input-output process. The input state is prepared in the state $|x, \theta\rangle$. After the transformation the operator $q_{\theta^{\prime}}$ is measured at the output. The statistics of this measurement are given by projection of the output state on $\left|x^{\prime}, \theta^{\prime}\right\rangle$, the eigenstates of $q_{\theta^{\prime}}$.

cillator [17]. The observables $q_{\theta}$ can be measured also in the case of trapped ions, as it is shown in Refs. $[5,6]$.

In this brief report we are interested in applying this technique to the characterization of arbitrary input-output transformations. To this end, we focus on the $s=s^{\prime}=0$ characteristic function $\tilde{\mathcal{U}}\left(\beta, 0 ; \beta^{\prime}, 0\right)$ with the aim of relating it to measurable quantities. After definitions (3.2) and (3.3) we have from Eq. (2.13):

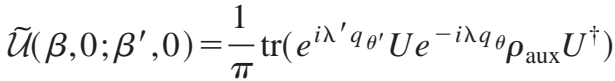

$$
\begin{aligned}
& =\frac{1}{\pi} \int d x^{\prime} d x e^{i\left(\lambda^{\prime} x^{\prime}-\lambda x\right)} P\left(x, \theta ; x^{\prime}, \theta^{\prime}\right),
\end{aligned}
$$

where

$$
P\left(x, \theta ; x^{\prime}, \theta^{\prime}\right)=\operatorname{tr}\left(\left|x^{\prime}, \theta^{\prime}\right\rangle\left\langle x^{\prime}, \theta^{\prime}|U| x, \theta\right\rangle\langle x, \theta| \rho_{\text {aux }} U^{\dagger}\right) .
$$

This implies that $\tilde{\mathcal{U}}\left(\beta, 0 ; \beta^{\prime}, 0\right)$ can be easily determined after obtaining from experiment the probabilities $P\left(x, \theta ; x^{\prime}, \theta^{\prime}\right)$. To this end, the input of the transformation must be prepared in the state $|x, \theta\rangle$ and the operator $q_{\theta^{\prime}}$ must be measured at the output, as schematized in Fig. 2. This demonstrates that the tomographic methods can be applied to the practical determination of arbitrary transformations.

Strictly speaking, the states $|x, \theta\rangle$ are not proper vectors of the Hilbert space $\mathcal{H}_{\text {in }}$, so in principle they cannot be generated. Nevertheless, it is possible to actually produce states as close as desired to $|x, \theta\rangle$. For example this is the case of squeezed states, which can be experimentally generated in quantum optics [18] as well as for the motion of trapped ions [19]. This approximation of rotated position or quadrature eigenstates by squeezed states was successfully considered before for the reconstruction of the motional state of a trapped ion in Ref. [6].

\section{TOMOGRAPHY IN LIOUVILLE SPACE}

Finally, we show that the tomography of states and transformations can be expressed by the same equations. This is possible by using the Liouville-space formulation where operators are represented by vectors in a suitably doubled Hilbert space [12]

$$
\left.\left.A=\sum_{n, m} A_{n m}|n\rangle\langle m|\leftrightarrow| A\rangle\right\rangle=\sum_{n, m} A_{n m}|n, m\rangle\right\rangle,
$$


where $|n\rangle$ is an orthonormal basis in the original Hilbert space, while $|n, m\rangle\rangle$ is the associated basis in the enlarged space. With this representation traces become scalar products

$$
\operatorname{tr}\left(A^{\dagger} B\right)=\langle\langle A \mid B\rangle\rangle .
$$

From Eqs. (2.3), (2.5), and (3.8) the basic function $P\left(x, \theta ; x^{\prime}, \theta^{\prime}\right)$ can be expressed as

$$
\begin{aligned}
P\left(x, \theta ; x^{\prime}, \theta^{\prime}\right) & =\sum_{m, k} p_{k}\left|\left\langle x^{\prime}, \theta^{\prime}\left|U_{m k}\right| x, \theta\right\rangle\right|^{2} \\
& =\sum_{m, k} p_{k}\left|\operatorname{tr}\left(|x, \theta\rangle\left\langle x^{\prime}, \theta^{\prime}\right| U_{m k}\right)\right|^{2} .
\end{aligned}
$$

Using Eq. (4.2) we arrive at the final expression

$$
P\left(x, \theta ; x^{\prime}, \theta^{\prime}\right)=\left\langle\left\langle x^{\prime}, \theta^{\prime} ; x, \theta\left|\rho_{\mathcal{U}}\right| x^{\prime}, \theta^{\prime} ; x, \theta\right\rangle\right\rangle,
$$

where $\left.\left|x^{\prime}, \theta^{\prime} ; x, \theta\right\rangle\right\rangle$ is defined by Eq. (4.1) as

$$
\left.\left|x^{\prime}, \theta^{\prime}\right\rangle\left\langle x, \theta|\leftrightarrow| x^{\prime}, \theta^{\prime} ; x, \theta\right\rangle\right\rangle
$$

and

$$
\left.\rho_{\mathcal{U}}=\sum_{m, k} p_{k}\left|U_{m k}\right\rangle\right\rangle\left\langle\left\langle U_{m k}\right|\right.
$$

is a true density matrix representing the transformation in Liouville space. It can be easily checked that $\rho_{\mathcal{U}} \geqslant 0, \rho_{\mathcal{U}}^{\dagger}$ $=\rho_{\mathcal{U}}$, and $\operatorname{tr} \rho_{\mathcal{U}}=1$.

Formally, Eq. (4.4) is exactly the same expression (3.5) applied to the quantum state represented by the density matrix $\rho_{\mathcal{U}}$ in the doubled Hilbert space. The vectors $\left.\left|x^{\prime}, \theta^{\prime} ; x, \theta\right\rangle\right\rangle$ are the eigenstates of rotated quadratures or rotated position in both Hilbert spaces of the enlarged space. The only difference with the standard tomography of quantum states is that in our case $\rho_{\mathcal{U}}$ describes transformations instead of states.

\section{CONCLUSIONS}

We have demonstrated the possibility of the tomographic determination of quantum input-output transformations. The formalism can be arranged so that formulas for the reconstruction of states and transformations are exactly the same. Therefore, the approach developed here can be regarded as an enlargement of quantum-state tomography to include more general objects.
[1] U. Leonhardt and H. Paul, Prog. Quantum Electron. 19, 89 (1995); U. Leonhardt, Measuring the Quantum State of Light (Cambridge University Press, Cambridge, England, 1997); D.-G. Welsch, W. Vogel, and T. Opatrný, in Progress in Optics, edited by E. Wolf (Elsevier Science, Amsterdam, 1999), Vol. 39.

[2] D. T. Smithey, M. Beck, M. G. Raymer, and A. Faridani, Phys. Rev. Lett. 70, 1244 (1993); S. Schiller, G. Breitenbach, S. F. Pereira, T. Müller, and J. Mlynek, ibid. 77, 2933 (1996); G. Breitenbach, S. Schiller, and J. Mlynek, Nature (London) 387, 471 (1997).

[3] D. Leibfried, D. M. Meekhof, B. E. King, C. Monroe, W. M. Itano, and D. J. Wineland, Phys. Rev. Lett. 77, 4281 (1996).

[4] M. Wilkens and P. Meystre, Phys. Rev. A 43, 3832 (1991); S. M. Dutra, P. L. Knight, and H. Moya-Cessa, ibid. 48, 3168 (1993); S. M. Dutra and P. L. Knight, ibid. 49, 1506 (1994); M. S. Kim, G. Antesberger, C. T. Bodendorf, and H. Walther, ibid. 58, R65 (1998); P. J. Bardroff, M. T. Fontenelle, and S. Stenholm, ibid. 59, R950 (1999); M. S. Kim and G. S. Agarwal, ibid. 59, 3044 (1999).

[5] S. Wallentowitz and W. Vogel, Phys. Rev. Lett. 75, 2932 (1995).

[6] J. F. Poyatos, R. Walser, J. I. Cirac, P. Zoller, and R. Blatt, Phys. Rev. A 53, R1966 (1996).

[7] J. F. Poyatos, J. I. Cirac, and P. Zoller, Phys. Rev. Lett. 78, 390 (1997); I. L. Chuang and M. A. Nielsen, J. Mod. Opt. 44, 2455 (1997); G. M. D’Ariano and L. Maccone, Phys. Rev. Lett. 80, 5465 (1998); V. Bužek, Phys. Rev. A 58, 1723 (1998).

[8] A. Luis and L. L. Sánchez-Soto, Phys. Lett. A 261, 12 (1999); Phys. Rev. Lett. 83, 3573 (1999).
[9] G. S. Agarwal and E. Wolf, Phys. Lett. A 26, 485 (1968); Phys. Rev. Lett. 21, 180 (1968); G. S. Agarwal, Phys. Rev. 177, 400 (1969); G. S. Agarwal and E. Wolf, Phys. Rev. D 2 , 2161 (1970); 2, 2187 (1970); 2, 2206 (1970).

[10] K. E. Cahill and R. J. Glauber, Phys. Rev. 177, 1857 (1969); 177, 1882 (1969); J. Peřina, Coherence of Light (D. Reidel, Dordrecht, 1985).

[11] K. Vogel and H. Risken, Phys. Rev. A 40, 2847 (1989).

[12] U. Fano, Rev. Mod. Phys. 29, 74 (1957); A. Royer, Phys. Rev. A 43, 44 (1991).

[13] C. W. Helstrom, Quantum Detection and Estimation Theory (Academic Press, New York, 1976); H. P. Yuen, Phys. Lett. A 91, 101 (1982).

[14] J. W. Noh, A. Fougères, and L. Mandel, Phys. Rev. Lett. 67, 1426 (1991); Phys. Rev. A 45, 424 (1992); 46, 2840 (1992); A. Luis and J. Peřina, Quantum Semiclassic. Opt. 8, 873 (1996).

[15] M. J. Bastiaans, Opt. Commun. 25, 26 (1978); G. García Calderón and M. Moshinsky, J. Phys. A 13, L185 (1980); R. Dirl, P. Kasperkovitz, and M. Moshinsky, ibid. 21, 1835 (1988); A. K. Ekert and P. L. Knight, Phys. Rev. A 43, 3934 (1991); A. Luis and L. L. Sánchez-Soto, Quantum Opt. 5, 33 (1993).

[16] S. Chountasis, A. Vourdas, and C. Bendjaballah, Phys. Rev. A 60, 3467 (1999).

[17] W. Vogel and D.-G. Welsch, Lectures on Quantum Optics (Akademie-Verlag, Berlin, 1994).

[18] J. Perina, Quantum Statistics of Linear and Nonlinear Optical Phenomena (Kluwer Academic, Dordrecht, 1991).

[19] J. I. Cirac, A. S. Parkins, R. Blatt, and P. Zoller, Phys. Rev. Lett. 70, 556 (1993); H. Zeng and F. Lin, Phys. Rev. A 52, 809 (1995). 